\title{
Photon momentum transfer in inhomogeneous dielectric mixtures and induced tractor beams
}

\author{
Cheng-Wei Qiu ${ }^{1,2}$, Weiqiang Ding ${ }^{3}$, M.R.C. Mahdy ${ }^{1}$, Dongliang Gao ${ }^{1}$, Tianhang Zhang ${ }^{1,2}$, Fook Chiong Cheong ${ }^{4}$, \\ Aristide Dogariu ${ }^{5}$, Zheng Wang ${ }^{6}$ and Chwee Teck Lim ${ }^{2,7}$
}

The determination of optical force as a consequence of momentum transfer is inevitably subject to the use of the proper momentum density and stress tensor. It is imperative and valuable to consider the intrinsic scheme of photon momentum transfer, particularly when a particle is embedded in a complex dielectric environment. Typically, we consider a particle submerged in an inhomogeneous background composed of different dielectric materials, excluding coherent illumination or hydrodynamic effects. A ray-tracing method is adopted to capture the direct process of momentum transfer from the complex background medium, and this approach is validated using the modified Einstein-Laub method, which uses only the interior fields of the particle in the calculation. In this way, debates regarding the calculation of the force with different stress tensors using exterior fields can be avoided. Our suggested interpretation supports only the Minkowski approach for the optical momentum transfer to the embedded scatterer while rejecting Peierls's and Abraham's approaches, though the momentum of a stably moving photon in a continuous background medium should be considered to be of the Abraham type. Our interpretation also provides a novel method of realizing a tractor beam for the exertion of negative force that offers an alternative to the use of negative-index materials, optical gain, or highly non-paraxial or multiple-light interference.

Light: Science \& Applications (2015) 4, e278; doi:10.1038/lsa.2015.51; published online 24 April 2015

Keywords: dielectric interface; Minkowski photon momentum transfer; modified Einstein-Laub method; optical pulling force; optical tractor beams

\section{INTRODUCTION}

Following the pioneering work of Marston' ${ }^{1}$ in acoustics, optical 'tractor beams' have attracted considerable interest by virtue of their unusual mechanism for micromanipulation. ${ }^{2-13}$ Generally speaking, a tractor beam is a customized light beam that exerts a negative scattering force (NSF) on a scatterer and pulls it opposite to the propagation direction of the light, in contrast to conventional pushing forces. ${ }^{14}$ Optical pulling forces provide a novel approach to gradientless optical manipulation techniques distinct from optical tweezers, ${ }^{15-17}$ optical conveyors ${ }^{13,18,19}$ and nanooptomechanical systems. ${ }^{20,21}$ Recently, various types of tractor beams have been experimentally demonstrated using a Gaussian beam with an optical mirror (involving the interference of incident and reflected light beams in certain limited regions $)^{8}$ and using dodecane droplets sitting on a dielectric interface. ${ }^{22}$ However, in the presence of a high-powered laser, hydrodynamic effects (uneven heat dissipation, particle absorption, temperature gradients, liquid convection, surface energy wells, etc.) may also contribute. Moreover, the stability criteria for tractor beams, which are very important for practical application, have not yet been investigated.

Although the mechanical effect has been demonstrated ${ }^{22}$ to be an overall consequence of all possible contributing factors, the mechanism of the optical momentum transfer from a mixed bi-medium background (air and water) to a partially submerged particle remains unclear, particularly regarding the issue of the proper stress tensor and force law to be adopted for non-vacuum backgrounds. Indeed, there are various forms of photon momentum, and various stress tensors, but why can only the Minkowski momentum be applied in this case? What stress tensor and force law should be adopted for nonvacuum and inhomogeneous backgrounds? These questions motivate us to investigate the fundamental physics governing the momentum transfer from light to a particle within an inhomogeneous (mixed) background medium, which invokes the Abraham-Minkowski controversy. ${ }^{23-30}$ In general, both Minkowskian ${ }^{24,28,29}$ and non-Minkowskian $^{10,30}$ formulations have been proposed for the measurement of the optical momentum transfer from a homogeneous background to an embedded particle. After a century of debate, it is not yet known which stress tensor (ST), force law and photon momentum scheme in matter are most appropriate to use in this scenario (e.g., Table I in Ref. 24). It appears that because the identification of an adequate 'particle momentum' is quite ambiguous and challenging, ${ }^{31}$ the recent resolution of the Abraham-Minkowski dilemma ${ }^{25}$ still cannot adequately

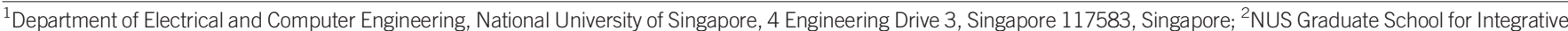

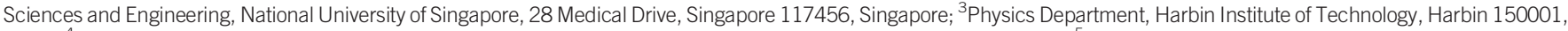

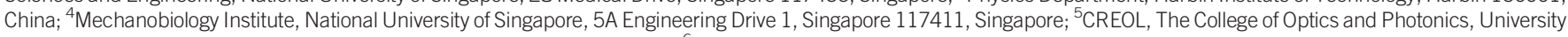

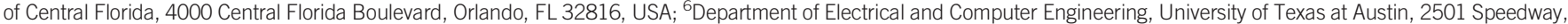
Austin, TX 78712, USA and ${ }^{7}$ Department of Mechanical Engineering, National University of Singapore, 9 Engineering Drive 1, Singapore 117576, Singapore

Correspondence: CW Qiu, Department of Electrical and Computer Engineering, National University of Singapore, E4-05-17, 4 Engineering Drive 3, Singapore 117583

E-mail: chengwei.qiu@nus.edu.sg

Received 27 July 2014; revised 24 January 2015; accepted 28 January 2015; accepted article preview online 29 January 2015 
address the question of the appropriate ST and force law to be employed in complex (mixed) backgrounds. ${ }^{31,32}$

In this context, it is valuable to pursue a simplified optical configuration without the limitations or implications discussed above, in which we can consider the mechanical light-matter interaction in isolation. For this purpose, we investigate the light momentum transfer and the related optical force on a transparent scatterer floating on a dielectric liquid-gas interface, ${ }^{33-35}$ as shown in Figure 1a. Although Webb et al. ${ }^{10,32}$ have proposed the Abraham photon momentum (APM) as the appropriate formulation for the purpose of the optical momentum transfer from a homogeneous background to an embedded particle, we demonstrate that in our case, it is more appropriate to consider the optical momentum transfer as a transfer of Minkowski photon momentum (MPM). Detailed calculations using the raytracing method and the modified Einstein-Laub equations (using the interior fields only) indicate that a negative pulling force and an optical tractor beam arise naturally in our scheme. Interestingly, nonMinkowskian formulations exhibit a pushing force, which contradicts the experimental observation reported in Ref. 22. However, although our suggested interpretation supports only the Minkowski approach for the process of optical momentum transfer to the embedded scatterer (near the scatterer and the dielectric interface), the photon momentum in a continuous background medium should be regarded as the Abraham type. Because this scheme can be extended to any gasliquid interface, we name our tractor beam an interfacial tractor beam'. The importance of this scheme is that it offers not only a practically achievable tractor beam, but also clear-cut insight based directly on the photon momentum, which is beneficial for practical
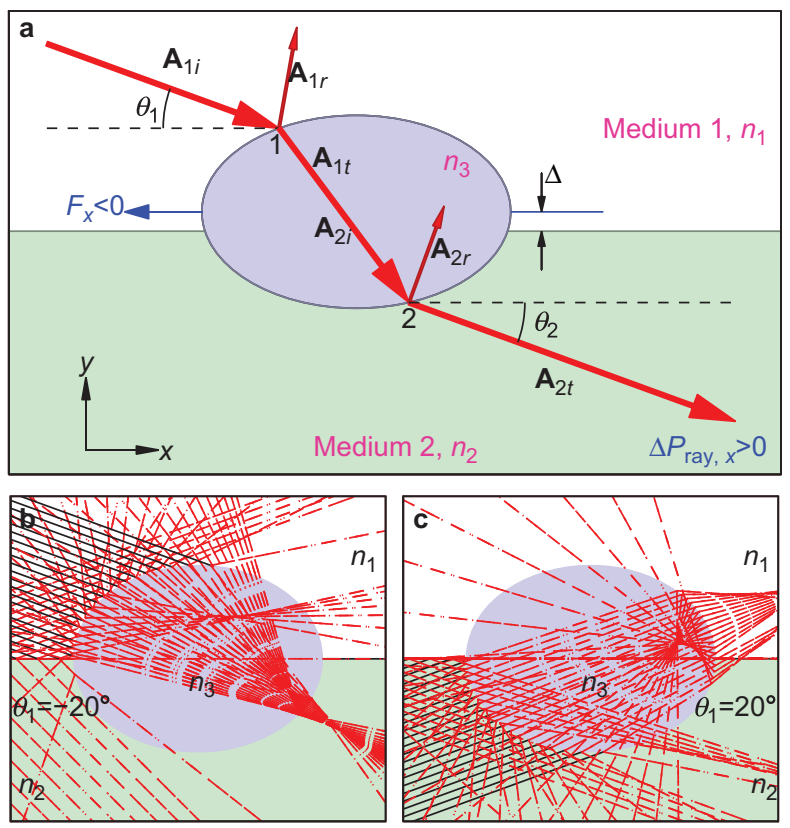

Figure 1 (a) A schematic diagram of photon momentum transfer in an inhomogeneous mixed background. $\mathbf{A}_{1 i, 1 r, 1 t}$ and $\mathbf{A}_{2 i, 2 r, 2 t}$ denote the propagation directions of the incident, reflected and transmitted rays at points 1 and 2 , respectively (the length of each arrow indicates $n_{m}$, the refractive index of the associated medium). $\theta_{1,2}$ describe the angles of the incident and transmitted rays with respect to the $+x$ axis. (b) The ray-tracing patterns of the system with $\theta_{1}=-20^{\circ}, r_{y}=0.75 r_{x}$ (where $r_{x}$ and $r_{y}$ are the semi-axes of the scatterer along the $x$ and $y$ directions, respectively), $n_{1}=1, n_{2}=1.33$ and $n_{3}=1.45$. The black (solid) and red (dashed) lines represent the incident and refracted rays, respectively. (c) The same as (b) except for $\theta_{1}=+20^{\circ}$. implementation. Of equal importance is our study of the stability criteria for such an interfacial tractor beam resulting from photon momentum transfer, which exhibit distinct behaviors from the optical-angular-momentum-based approach. ${ }^{36}$

\section{MATERIALS AND METHODS}

\section{The ray-tracing method and the Minkowski stress tensor,} employing background fields

The proposed background mixture is illustrated in Figure 1a. The scatterer (with a refractive index of $n_{3}$ ) is suspended at the interface of a liquid (e.g., water, with a refractive index of $n_{2}$ ) and a gas (e.g., air, $n_{1}$ ). The incident and scattered beams may lie in different media (also shown in the ray-tracing patterns of Figure $1 \mathrm{~b}$ and $1 \mathrm{c}$ ). Therefore, not only the direction but also the amplitude of the momentum of the light is changed. Without loss of generality, we restrict the calculation to two-dimensional (2D) structures for clarity. Generally, an enclosed integration path should be selected outside the scatterer for the calculation of the optical force. However, there is ambiguity in this method in the current scenario because the integration path must pass through different media (the gas and the liquid). To avoid this ambiguity, a ray-tracing method is developed for the force calculation.

In the ray-tracing method (see Section 1 in Supplementary Information for details), the momentum of a photon in each medium should first be clearly defined. Generally, the momenta $p_{1,2}$ carried by a photon in media 1 and 2, respectively, are

$$
p_{1}=\alpha_{1} p_{0}=\alpha_{1} \frac{\hbar \omega}{c}, p_{2}=\alpha_{2} p_{0}=\alpha_{2} \frac{\hbar \omega}{c}
$$

where $\hbar, \omega$ and $c$ are the reduced Planck constant, the angular frequency, and the speed of light in vacuum, respectively. $p_{0}=\hbar \omega / c$ is the momentum of the photon in vacuum. For the material-related constants $\alpha_{1,2}$; however, different values have been proposed, ${ }^{24,27,29}$ such as $\alpha=n$ for the Minkowski formula, ${ }^{24,27,29} \alpha=n^{-1}$ for the Abraham formula ${ }^{24,27,29}$ and $\alpha=\left(4 n+7 n^{2}-n^{4}\right) /(10 n)$ for the Peierls formula, ${ }^{37,38}$ and there is long-standing controversy on this issue. Although it is believed that this controversy was resolved in Ref. 25, the exchange of momentum between light and matter, and therefore, the forces exerted on objects, is still dependent on the particularities of the situation. ${ }^{24,27,29}$ Experimental results suggest that when an interface is present, the Minkowski formula should be used to correctly obtain the related optical force $^{14}$ (see Section 2 in Supplementary Information for details).

As illustrated in Figure 1a, the changes in the momentum of the light rays from air to water through the scatterer itself can be calculated by summing up the momentum change associated with each refraction. As a close approximation, only two refractions, corresponding to $\Delta \mathbf{P}_{\text {ray }}=\Delta \mathbf{P}_{\text {ray }, 1}+\Delta \mathbf{P}_{\text {ray }, 2}$ (see Section 1 in Supplementary Information for details), are considered: $\Delta \mathbf{P}_{\text {ray }, 1}=P_{0}\left(T_{1} \mathbf{A}_{1 t}+R_{1} \mathbf{A}_{1 r}-\mathbf{A}_{1 i}\right)$ and $\Delta \mathbf{P}_{\text {ray }, 2}=P_{0}\left(T_{1} T_{2} \mathbf{A}_{2 t}+T_{1} R_{2} \mathbf{A}_{2 r}-T_{1} \mathbf{A}_{1 t}\right)$. Here, $P_{0}$ is the amplitude of the momentum of the incident ray. $\mathbf{A}_{1 i, 1 t, 1 r}$ and $\mathbf{A}_{2 i, 2 t, 2 r}$ are the directional vectors along the incident, transmitted, and reflected rays for the first and second refractions, ${ }^{39}$ respectively. $T_{1,2}$ and $R_{1,2}$ are the energy transmission and reflection coefficients determined by the Fresnel formula. For the special case of $\theta_{1}=0$ and sufficiently small $R_{1,2}$ (e.g., $R_{1}=3.4 \%$ and $R_{2}=0.2 \%$ at $\theta_{1}=0$ for the parameters given in Figure 1), one can directly obtain $\Delta P_{\text {ray }, x} / P_{0}=T_{1} T_{2} n_{2} \cos \theta_{2}-n_{1}$. In the usual case of light scattering in a homogenous background $\left(n_{1}=n_{2}\right)$, $\Delta P_{\text {ray }, x}$ is certainly less than zero. However, for the inhomogeneous mixture background depicted in Figure 1a, the condition $n_{2}>n_{1} /$ $\left(T_{1} T_{2} \cos \theta_{2}\right)$ may easily be satisfied, thereby resulting in a positive 
$\Delta P_{\text {ray, } x}$. Thus, an induced negative force on the submerged object is a natural consequence of linear momentum conservation.

In the analysis presented above, no restrictions (beam shape, polarization, relative phase, coherence, etc.) on the optical fields are imposed; only the law of conservation of linear momentum is invoked. Therefore, a simple optical field, such as a single plane wave, can be used to realize an NSF in practice. We attribute the greatly simplified conditions required to achieve an NSF in our scheme to the presence of the liquid, which provides a channel to increase the forward momentum in a passive environment. Previously reported NSF scen$\operatorname{arios}^{2-13}$ have included only a light beam and a scatterer, and the background medium has been uniform and contributed equally to both the incident and scattered beams. As proposed in Ref. 40 and demonstrated in Ref. 22, the background medium also plays an important role in the generation of a tractor beam. To obtain a pulling force with a structured background as in Ref. 22, however, the hydrodynamic effect, a convection current and uneven heating all must be present. By contrast, in our scenario, it can be observed that even if such effects are omitted, it is possible to change the magnitude of the optical pulling force simply by modifying the frequency of the light source, the difference in the refractive indexes of the two background media (the refractive index of the final background medium should be higher than that of the previous medium (or media) to achieve optical pulling), and the shape or size of the submerged particle.

To calculate the momentum transfer and the corresponding forces quantitatively, we set the media of the gas, the liquid and the scatterer to be, respectively, air, water and silica sphere with indexes of $n_{1}=1.0$, $n_{2}=1.33$ and $n_{3}=1.45$. Using the ray-tracing method mentioned above, all rays impinging on the scatterer are traced (as illustrated in Figure $1 \mathrm{~b}$ and $1 \mathrm{c}$ ), and the momentum changes and, in turn, the optical force are calculated correspondingly. Figure 2a shows the changes in $F_{x}$ with the shape of the scatterer for the case of $\theta_{1}=0^{\circ}$. When $0.425<r_{y} / r_{x}<0.775$, both $p$ and $s$ polarizations can produce a negative force. Figure $2 \mathrm{~d}$ shows the changes in the force with the angle of incidence on a circular scatterer with $r_{x}=r_{y}$, and NSFs are achieved within a broad range of incidence directions. These results are consistent with the analysis presented above.

To calculate the force using STs, we calculate the scattering using the finite-difference time-domain method for a plane wave incident on a circular scatterer (with semi-axes along the $x$ direction, $r_{x}$, and along the $y$ direction, $r_{y}$, of $r_{x}=r_{y}=2.0 \mu \mathrm{m}$ ) and an elliptical scatterer (with $r_{x}=2 \mu \mathrm{m}$ and $r_{y}=0.4 \mu \mathrm{m}$ ). Then, the optical forces experienced by the particles are calculated through the integration of the time-averaged Minkowski stress tensor on a closed contour (see the dashed rectangles in Figure $2 \mathrm{c}$ and $2 \mathrm{f}$ ) surrounding the scatterer,

$$
\langle\bar{T}\rangle=\frac{1}{2} \operatorname{Re}\left[\mathbf{D E}^{*}+\mathbf{B H}^{*}-\frac{1}{2} \mathbf{I}\left(\mathbf{E}^{*} \cdot \mathbf{D}+\mathbf{H}^{*} \cdot \mathbf{B}\right)\right]
$$

The calculated forces for the elliptical and circular scatterers are presented in Figure 2b and 2e (square lines), respectively, and good agreement with the results of the ray-tracing method is observed. Here, the s-polarization results are not shown because they are similar to those of the $p$-polarization case. In the calculation, the local permittivity and permeability along the integration paths are used (as shown in Figure $2 \mathrm{c}$ and $2 \mathrm{f}$ ). Figure $2 \mathrm{c}$ and $2 \mathrm{f}$ present the field patterns of $H_{z}$ for the scatterer in the case of $\theta_{1}=-30^{\circ}$ for the circular and elliptical scatterers, respectively. The arrows represent the elementary force vectors on the closed contour along which the total net force $F_{x}$ is calculated via integration. The majority of the pulling force is exerted when the photons leave the scatterer, consistent with our analysis.

\section{Explanation of the observations and two-photon momenta}

It is important to note that our previous analysis and the experimental observation reported in Ref. 22 cannot distinguish whether the momentum of a photon moving in a continuous background (water) is the Minkowski momentum. In a similar previous experiment (the well-known Jones experiment), optical-force-formula-based approaches supported the transfer of both the MPM ${ }^{31}$ and the APM. ${ }^{32}$ Such ambiguities regarding the transfer of photon momentum from the background can only be overcome via a direct photon momentum approach $^{24}$ such as the ray-tracing method, which was the primary focus of the previous section. Surprisingly, for the experiment presented in Ref. 22, the force experienced by the embedding water medium should be calculated using the Einstein-Laub formula based on the field inside the water, which indeed supports the APM formulation (cf. Equation (21) in Refs. 32 and 41). If the force on a lossless continuous medium is calculated via the Minkowski ST (associated with the MPM) employing the interior fields, the calculation will yield zero force. In the cited experiment, ${ }^{22}$ at the interface of the scatterer and the water, ${ }^{22}$ the MPM should arise because of the reduced impedance mismatch. ${ }^{42}$ In fact, the MPM generates a translation of the electromagnetic field. ${ }^{26}$ This translation relative to the host is exactly the quantity required to represent the displacement of an embedded object. ${ }^{25}$ As a result, the MPM appears in almost all major radiation pressure experiments that measure the displacement of an embedded object. ${ }^{25,26}$ However, after some duration of photon emission by the scatterer, the dressed photon (MPM) should start to lose its momentum, slowly but continuously. That momentum should be absorbed by the water medium, and finally, the momentum of the moving photon should be the APM. In this discussion, it is important to mention the experimental observations of Ashkin and Dziedzic regarding the force density distribution in water, ${ }^{33}$ which support the Einstein-Laub forcedensity expression associated with the APM, according to Mansuripur et al. ${ }^{41}$ Therefore, the experimental observation of a pulling force on the scatterer in Ref. 22 indeed cannot prove that the MPM is the only correct photon momentum. In fact, both the MPM and the APM are correct, but their functionalities are quite different.

\section{The validity of other methods}

Hitherto, the transfer of optical momentum has been calculated based on the MPM approach, which is also in good agreement with the experimental observations reported in Ref. 22. Surprisingly, no other photon momentum (Abraham or Peierls) predicts an optical pulling force (see Supplementary Fig. S1 in Section 2 in Supplementary Information). In addition, the force calculation results based on the Lorentz formula and the Chu formula that are presented in Figure $2 \mathrm{~b}$ and $2 \mathrm{e}$, which are calculated via the integration of the Lorentz force density (from the interior of the embedded particle) and via Chu's ST (from the exterior of the embedded particle), ${ }^{24}$ do not guarantee negative forces (the Nelson ${ }^{43}$ stress tensor leads to the same result). In Ref. 29, it was considered that the MPM bears a momentum contribution from the single-photon momentum (field momentum or Abraham momentum ${ }^{24,29}$ ) along with a momentum contribution from the material, ${ }^{24} \boldsymbol{P}_{\text {med. }}{ }^{29}$ Although Milonni and Boyd ${ }^{29}$ have considered that the momentum of the medium $\left(\boldsymbol{P}_{\text {med }}\right)$ should be regarded as the momentum that is shared with the background medium (or host ${ }^{26}$ ) by the field, we argue, likely for the first time, that $\boldsymbol{P}_{\text {med }}$ should be regarded only as a transfer of mechanical momentum from the 

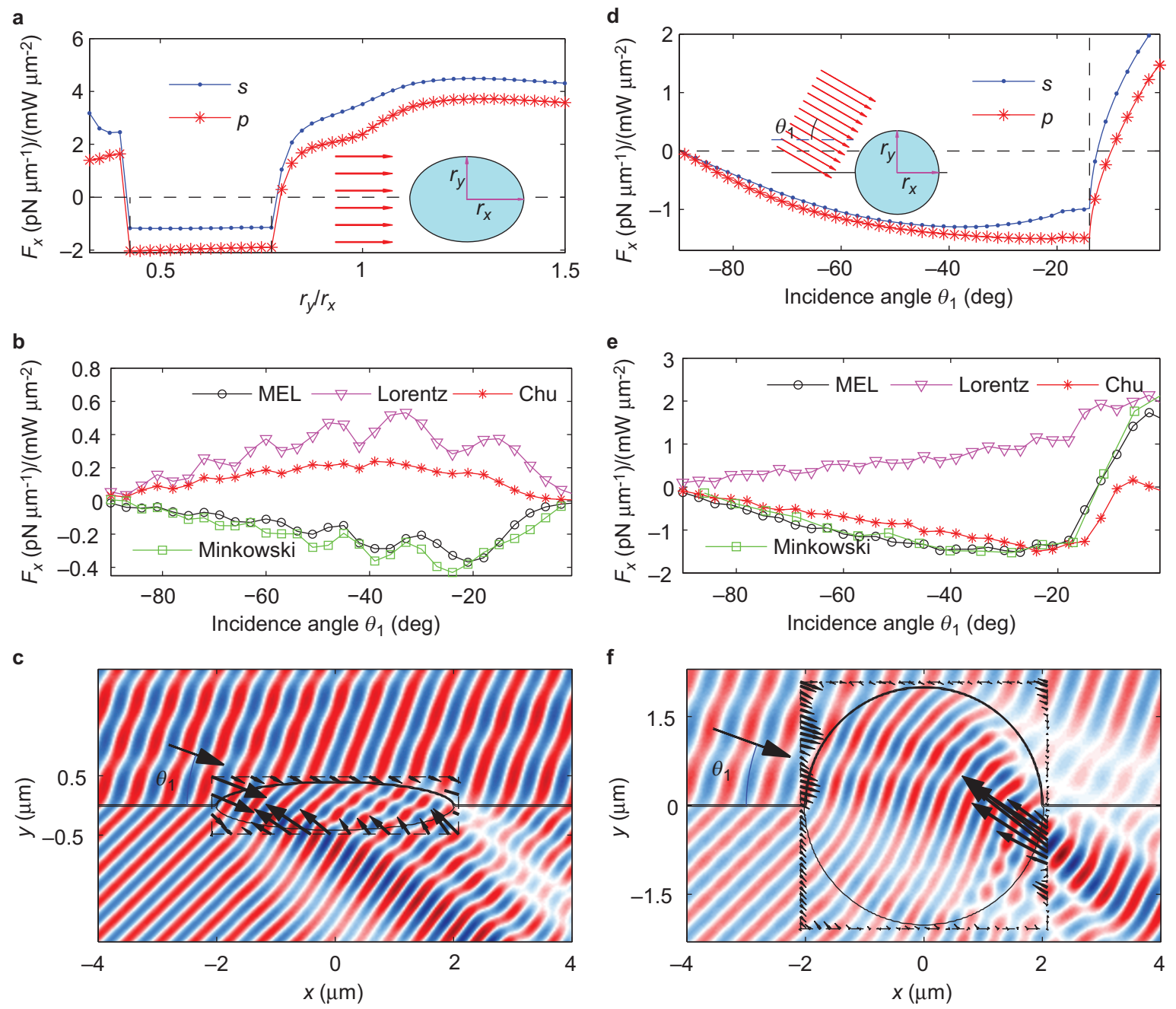

Figure 2 Optical forces derived using different formulas. The parameters are $n_{1}=1.0, n_{2}=1.33, n_{3}=1.45$ and $\Delta=0$, unless otherwise specified. (a) The variation in the optical forces with the shape $r_{y} r_{x}$ at $\theta_{1}=0$. The forces are calculated using the ray-tracing method, in which the Minkowski approach is adopted for the photon momentum. (b) The variation in the optical forces with the incident direction $\theta_{1}$ for the $p$-polarization case, as calculated via the modified Einstein-Laub formula (MEL, circle line) proposed in this paper, volume integration of the Lorentz force density (Lorentz, triangle line), the Chu formula (Chu, asterisk line) and the Minkowski stress tensor (Minkowski, square line). The size of the scatterer is defined by $r_{x}=2.0 \mu \mathrm{m}$ and $r_{y}=0.4 \mu \mathrm{m}$ (as shown in (c)). (c) The field pattern for $H_{z}$ in the case of $\theta_{1}=-30^{\circ}$. (d) The variation in the optical forces with the incident direction $\theta_{1}$ for a circular scatterer of $r_{x}=r_{y}=2.0 \mu \mathrm{m}$. The forces are calculated using the ray-tracing method. (e, f) The same as (b) and (c), respectively, except for $r_{y}=2.0 \mu \mathrm{m}$.

background to the embedded particle (see also Supplementary Fig. S1 in Section 2 and, later, Section 3 in Supplementary Information).

According to Milonni and Boyd, ${ }^{29}$ this $\boldsymbol{P}_{\text {med }}$ originates from the extra time-varying Abraham term $\boldsymbol{f}^{A}$. However, the time average of $\boldsymbol{f}^{A}$ becomes zero when we consider the time-averaged total force or the time-averaged momentum transfer. Our proposal also explains why the Helmholtz force term, $f_{H}$, in the linear momentum conservation formula of Minkowski ${ }^{44,45}$ fails to predict the total time-averaged mechanical force without the addition of hidden terms from elasticity and thermodyn$\operatorname{amics}^{44,45}$ (also see Section 3 in Supplementary Information).

Because the time-varying Minkowski momentum density (or the MPM) carries the mechanical momentum component, the time-averaged force calculated using the associated Helmholtz force fails to predict the correct total mechanical force. Moreover, the Abraham force law also fails to yield the total force, as $f^{A}$ becomes zero when the time average is taken. As a result, from our point of view, the raytracing methods based on the MPM presented in this article or the Minkowski stress tensor based on the background fields should be employed to calculate the correct result (see the three-dimensional cases presented in Section 4 in Supplementary Information). Although the Chu stress tensor based on the exterior fields exhibits an optical pulling force for the case of a 2D circle (Figure 2e), it exhibits a pushing force for the 2D ellipse (Figure $2 \mathrm{~b}$ ). In fact, the optical momentum transfer from the background to an embedded particle should not be calculated using the Chu or Nelson stress tensor. For non-magnetic media, the momentum density associated with these formulas is the Abraham momentum. As a result, the Chu and Nelson formulations fail to account for the effect of the transfer of the mechanical momentum $\boldsymbol{P}_{\text {med }}$ (the additional backward momentum). Although several force and ST formulations have been discussed in Ref. 46 in an attempt to explain various experimental observations (other than those obtained for dielectric mixtures), the problems associated with the different photon momenta have not been clarified. In this article, even for a very complex scenario, we have attempted to resolve the problems of both STs and the associated photon momenta. 


\section{RESULTS AND DISCUSSION}

Modified Einstein-Laub stress tensor based on the interior fields of the scatterer

Another method of unambiguously calculating the optical force is to use the force density inside the scatterer rather than the fields outside it. Surprisingly, the time-averaged Lorentz force obtained by employing the interior fields ${ }^{47}$ of the embedded particle also fails to yield an accurate result for the total mechanical force, as shown in Figure $2 b$ and 2e. The Lorentz force reported in Ref. 47 (actually the internal Chu force for non-magnetic media ${ }^{24}$ ) based on the interior fields and the APM is applicable only when the background medium is air. It is for this reason that the interior of a particle can be modeled as the interior of the conventional Einstein-Balaz box experiment ${ }^{29}$ (the photon momentum is the Abraham momentum in the interior of the box $^{26,29}$ ) only when the background is air. Therefore, conclusions based on the Einstein-Balaz box experiment do not remain valid when the background is not air and an additional mechanical momentum $\boldsymbol{P}_{\text {med }}$ is transferred via the MPM from the external background (i.e., the origin of the additional pulling force after the emission of the photon by the scatterer in Ref. 22). If the background medium is not air, then the Lorentz force ${ }^{47}$ on the interior of the embedded particle should be modified. From our point of view, the interior mechanical force should be calculated via the time-averaged modified Einstein-Laub (MEL) stress tensor: ${ }^{48}$

$$
\mathbf{F}_{\text {Total }}(\text { from interior fields })=\frac{1}{2} \operatorname{Re}\left[\oint\left\langle\overline{\boldsymbol{T}}_{\mathrm{MEL}}(\text { in })\right\rangle \cdot \mathrm{d} \mathbf{S}\right]
$$

where $\left\langle\overline{\boldsymbol{T}}_{\text {MEL }}(\right.$ in $\left.)\right\rangle=\mathbf{D}_{i n} \mathbf{E}_{i n}^{*}+\mathbf{B}_{i n} \mathbf{H}_{i n}^{*}-\frac{1}{2}\left(\mu_{b} \mathbf{H}_{i n}^{2}+\varepsilon_{b} \mathbf{E}_{i n}^{2}\right) \overline{\mathbf{I}}, \mu_{b}$ and $\varepsilon_{b}$ are the permeability and permittivity of the background medium of the particle, and $\overline{\mathbf{I}}$ is the unit tensor. In our setup, the permeability and permittivity of the background are the local permeability and permittivity of the air and water, respectively, defined in the specific $2 \mathrm{D}$ configuration of the full-wave simulation (Figure $2 \mathrm{~b}$ and $2 \mathrm{e}$ ). The quantities $\mathbf{E}_{\text {in }}, \mathbf{H}_{\text {in }}, \mathbf{D}_{\text {in }}$ and $\mathbf{B}_{\text {in }}$ refer to the values in the interior of the silica particle. Note that no hidden quantity is required to obtain the modified Einstein-Laub stress tensor. The calculations via the MEL stress tensor based on the interior fields are in full agreement with the ray-tracing method and Minkowski formulations based on the exterior fields (Figure $2 \mathrm{~b}$ and 2e). Detailed discussions of MEL formulations ${ }^{48}$ will be presented in our future articles.

\section{The dependence of the NSF on the vertical distance of the particle from the interface}

Also of interest is the stable dependence of the interfacial tractor beam on the vertical distance $\Delta$ of the particle from the interface (as labeled in Figure 1a). The results show that the NSF is robust with respect to the vertical position $\Delta$, as illustrated in Figure 3. However, the surface energy well at the water-air interface can stably trap the scatterer in the vertical direction (see Section 5 in Supplementary Information for details). In the tangential direction, the motion equation is

$$
m \frac{\mathrm{d} v}{\mathrm{~d} t}=F_{\mathrm{scat}}-F_{\mathrm{d}}=F_{\mathrm{scat}}-3 \pi \mu r \cdot v
$$

where $m$ and $v$ are the mass and velocity of the object. $F_{\text {scat }}$ and $F_{\mathrm{d}}=3 \pi \mu r \cdot v$ are the optical scattering force and the drag force of the water, respectively. Then, the final velocity can be calculated by setting $\mathrm{d} v / \mathrm{d} t=0$, i.e., $v_{\text {final }}=F_{\text {scat }} / 3 \pi \mu r$. Suppose that the temperature is $300 \mathrm{~K}$; in that case, the dynamic viscosity of water is approximately $\mu \approx 8 \times 10^{-4} \mathrm{~Pa} \mathrm{~s}^{49}$ Then, a linear relation between the velocity and the optical scattering force can be obtained.

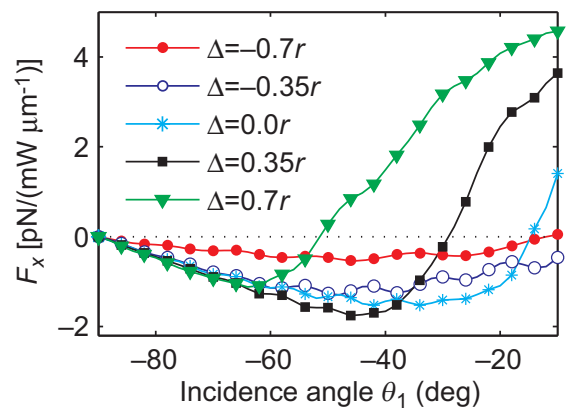

Figure 3 Optical forces calculated using the ray-tracing method when the center of the scatterer is located at a distance $\Delta$ (as labeled in Figure 1a) from the liquidgas interface. The incident light is a $p$-polarized plane wave, and the object is circular with a radius of $r_{x}=r_{y}=2 \mu \mathrm{m}$. All other parameters are the same as those in Figure 2.

\section{CONCLUSIONS}

In summary, we demonstrated that the transfer of photon momentum from an inhomogeneous background with a single submerged particle supports the Minkowski formulation and that increased forward momentum results from a bi-background medium configuration, in which the particle plays an important role. The problems of how the field, the mechanical momentum, the optical force and the stability of the negative force are related to the momentum transfer process were unambiguously addressed. The optical scattering force can thus be robustly adjusted (positive or negative) using the correct momentum transfer scheme. As a result, an interfacial tractor beam can be stably realized over a wide range of incidence angles and vertical positions of transparent particles. We believe that our proposal is very effective in resolving not only the Abraham-Minkowski photon momentum dilemma, but also the question of the associated stress tensors and optical forces, including tractor beams.

\section{ACKNOWLEDGEMENTS}

CWQ acknowledges financial support from the National University of Singapore (Grant No. R-263-000-678-133). ZW acknowledges support from the Packard Fellowship in Science and Engineering and the Alfred P. Sloan Research Fellowship. CWQ and WD contributed equally.

1 Marston PL. Axial radiation force of a Bessel beam on a sphere and direction reversal of the force. J Acoust Soc Am 2006; 120: 3518-3524.

2 Sukhov S, Dogariu A. On the concept of "tractor beams". Opt Lett 2010; 35: 3847 3849.

3 Chen J, Ng J, Lin Z, Chan CT. Optical pulling force. Nat photonics 2011; 5: 531-534.

4 Novitsky A, Qiu CW, Wang H. Single gradientless light beam drags particles as tractor beams. Phys Rev Lett 2011; 107: 203601.

5 Novitsky A, Qu CW, Lavrinenko A. Material-independent and size-independent tractor beams for dipole objects. Phys Rev Lett 2012; 109: 023902.

6 Lee SH, Roichman Y, Grier DG. Optical solenoid beams. Opt Express 2010; 18: 6988-6993.

7 Sukhov S, Dogariu A. Negative nonconservative forces: optical "tractor beams" for arbitrary objects. Phys Rev Lett 2011; 107: 203602.

8 Brzobohatý O, Karásek V, Šiler M, Chvátal L, Čižmár T et al. Experimental demonstration of optical transport, sorting and self-arrangement using a 'tractor beam'. Nat Photonics 2013; 7: 123-127.

9 Mizrahi A, Fainman Y. Negative radiation pressure on gain medium structures. Opt Lett 2010; 35: 3405-3407.

10 Webb KJ, Shivanand K. Negative electromagnetic plane-wave force in gain media. Phys Rev E 2011; 84: 057602.

11 Salandrino A, Christodoulides DN. Reverse optical forces in negative index dielectric waveguide arrays. Opt Lett 2011; 36: 3103-3105.

12 Nieto-Vesperinas M, Sáenz JJ, Gómez-Medina R, Chantada L. Optical forces on small magnetodielectric particles. Opt Express 2010; 18: 11428-11443. 
13 Ruffner DB, Grier DG. Optical conveyors: a class of active tractor beams. Phys Rev Lett 2012; 109: 163903.

14 Swartzlander GA, Peterson TJ, Artusio-Glimpse AB, Raisanen AD. Stable optical lift. Nat Photonics 2011; 5: 48-51.

15 Ashkin A. Acceleration and trapping of particles by radiation pressure. Phys Rev Lett 1970; 24: 156-159.

16 Ashkin A, Dziedzic JM, Bjorkholm JE, Chu S. Observation of a single-beam gradient force optical trap for dielectric particles. Opt Lett 1986; 11: 288-290.

17 Moffitt JR, Chemla YR, Smith SB, Bustamante C. Recent advances in optical tweezers. Annu Rev Biochem 2008; 77: 205-228.

18 Miller PD, Akhmediev NN, Ankiewicz A. Optical conveyor belts: a new scheme for fiber communications. Opt Lett 1996; 21: 1132-1134.

19 Čižmár T, Garcés-Chávez V, Dholakia K, Zemánek P. Optical conveyor belt for delivery of submicron objects. Appl Phys Lett 2005; 86: 174101.

20 Li M, Pernice WH, Tang HX. Tunable bipolar optical interactions between guided lightwaves. Nat Photonics 2009; 3: 464-468.

21 Wiederhecker GS, Chen L, Gondarenko A, Lipson M. Controlling photonic structures using optical forces. Nature 2009; 462: 633-636.

22 Kajorndejnukul V, Ding W, Sukhov S, Qiu CW, Dogariu A. Linear momentum increase and negative optical forces at dielectric interface. Nat Photonics 2013; 7: 787-790.

23 Mansuripur M. Trouble with the Lorentz law of force: incompatibility with special relativity and momentum conservation. Phys Rev Lett 2012; 108: 193901.

24 Kemp BA. Resolution of the Abraham-Minkowski debate: implications for the electromagnetic wave theory of light in matter. J App/ Phys 2011; 109: 111101.

25 Barnett SM. Resolution of the Abraham-Minkowski dilemma. Phys Rev Lett 2010; 104: 070401.

26 Barnett SM, Loudon R. The enigma of optical momentum in a medium. Philos Trans $R$ Soc A 2010; 368: 927-939.

27 Baxter C, Loudon R. Radiation pressure and photon momentum in dielectrics. J Mod Opt 2010; 57: 830-842.

28 Loudon R, Barnett SM, Baxter C. Radiation pressure and momentum transfer in dielectrics: the photon drag effect. Phys Rev A 2005; 71: 063802.

29 Milonni PW, Boyd RW. Momentum of light in a dielectric medium. Adv Opt Photonics 2010; 2: 519-553.

30 Pfeifer RN, Nieminen TA, Heckenberg NR, Rubinsztein-Dunlop H. Colloquium: momentum of an electromagnetic wave in dielectric media. Rev Mod Phys 2007; 79: 1197-1216.

31 Griffiths DJ. Resource letter EM-1: electromagnetic momentum. Am J Phys 2012; 80: 7-18.

32 Webb KJ. Dependence of the radiation pressure on the background refractive index. Phys Rev Lett 2013; 111: 043602.
33 Ashkin A, Dziedzic JM. Radiation pressure on a free liquid surface. Phys Rev Lett 1973; 30: 139-142.

34 Brasselet E, Wunenburger R, Delville JP. Liquid optical fibers with a multistable core actuated by light radiation pressure. Phys Rev Lett 2008; 101: 014501.

35 Emile 0, Emile J. Low-power laser deformation of an air-liquid interface. Phys Rev Lett 2011; 106: 183904.

36 Wang N, Chen J, Liu S, Lin Z. Dynamical and phase-diagram study on stable optical pulling force in Bessel beams. Phys Rev A 2013; 87: 063812.

37 Peierls R. The momentum of light in a refracting medium. Proc $R$ Soc Lond Ser $A$ 1976; 347: 475-491.

38 Peierls R. The momentum of light in a refracting medium. II. Generalization. Application to oblique reflexion. Proc $R$ Soc Lond Ser A 1977; 355: 141-151.

39 Tkaczyk ER. Vectorial laws of refraction and reflection using the cross product and dot product. Opt Lett 2012; 37: 972-974.

40 Dogariu A, Sukhov S, Sáenz JJ. Optically induced 'negative forces'. Nat Photonics 2013; 7: 24-27.

41 Mansuripur M, Zakharian AR, Wright EM. Electromagnetic-force distribution inside matter. Phys Rev A 2013; 88: 023826.

42 Mansuripur M, Zakharian AR. Whence the Minkowski momentum? Opt Commun 2010; 283: 3557-3563.

43 Loudon R, Allen L, Nelson DF. Propagation of electromagnetic energy and momentum through an absorbing dielectric. Phys Rev E 1997; 55: 1071-1085.

44 Mansuripur M. The force law of classical electrodynamics: Lorentz versus Einstein and Laub. Proc SPIE 2013; 8810: 88100K

45 Lai HM, Suen WM, Young K. Microscopic derivation of the Helmholtz force density. Phys Rev Lett 1981; 47: 177-179.

46 Brevik I. Experiments in phenomenological electrodynamics and the electromagnetic energy-momentum tensor. Phys Rep 1979; 52: 133-201.

47 Kemp BA, Grzegorczyk TM, Kong JA. Optical momentum transfer to absorbing Mie particles. Phys Rev Lett 2006; 97: 133902.

48 Mahdy MRC. It should be Einstein-Laub equations inside matter. arXiv: 1211.0155 2012.

49 Viscosity. Available at http://en.wikipedia.org/wiki/Viscosity.

(i) (2) This work is licensed under a Creative Commons Attributioncc) NG SA NonCommercial-ShareAlike 3.0 Unported License. The images or other third party material in this article are included in the article's Creative Commons license, unless indicated otherwise in the credit line; if the material is not included under the Creative Commons license, users will need to obtain permission from the license holder to reproduce the material. To view a copy of this license, visit http://creativecommons.org/licenses/by-nc-sa/3.0/ 